\title{
Inhibition of VEGF receptors causes lung cell apoptosis and emphysema
}

\author{
Yasunori Kasahara, ${ }^{1}$ Rubin M. Tuder, ${ }^{1,2}$ Laimute Taraseviciene-Stewart ${ }^{2}$ \\ Timothy D. Le Cras, ${ }^{3}$ Steven Abman, ${ }^{3}$ Peter K. Hirth, ${ }^{4}$ Johannes Waltenberger, ${ }^{5}$ \\ and Norbert F. Voelkel ${ }^{1}$
}
${ }^{1}$ Division of Pulmonary Sciences and Critical Care Medicine, Pulmonary Hypertension Center, Department of Medicine,
${ }^{2}$ Department of Pathology, and
${ }^{3}$ Pediatric Heart Lung Center, Division of Pediatric Pulmonary Medicine, Department of Pediatrics,
University of Colorado Health Sciences Center, Denver, Colorado, USA
${ }^{4}$ SUGEN Inc., South San Francisco, California, USA
${ }^{5}$ Department of Internal Medicine II, Division of Cardiology, Ulm University Medical Center, Ulm, Germany
Address correspondence to: Norbert F. Voelkel, Division of Pulmonary Sciences and Critical Care Medicine, University of Colorado Health Sciences Center, Box C 272, 4200 E. Ninth Avenue, Denver, Colorado 80262, USA. Phone: (303) 315-7047; Fax: (303) 315-5632; E-mail: Norbert.Voelkel@UCHSC.edu.

Received for publication May 8, 2000, and accepted in revised form August 30, 2000.

\begin{abstract}
Pulmonary emphysema, a significant global health problem, is characterized by a loss of alveolar structures. Because VEGF is a trophic factor required for the survival of endothelial cells and is abundantly expressed in the lung, we hypothesized that chronic blockade of VEGF receptors could induce alveolar cell apoptosis and emphysema. Chronic treatment of rats with the VEGF receptor blocker SU5416 led to enlargement of the air spaces, indicative of emphysema. The VEGF receptor inhibitor SU5416 induced alveolar septal cell apoptosis but did not inhibit lung cell proliferation. Viewed by angiography, SU5416-treated rat lungs showed a pruning of the pulmonary arterial tree, although we observed no lung infiltration by inflammatory cells or fibrosis. SU5416 treatment led to a decrease in lung expression of VEGF receptor 2 (VEGFR-2), phosphorylated VEGFR-2, and Akt-1 in the complex with VEGFR-2. Treatment with the caspase inhibitor Z-Asp- $\mathrm{CH}_{2}-\mathrm{DCB}$ prevented SU5416-induced septal cell apoptosis and emphysema development. These findings suggest that VEGF receptor signaling is required for maintenance of the alveolar structures and, further, that alveolar septal cell apoptosis contributes to the pathogenesis of emphysema.
\end{abstract}

J. Clin. Invest. 106:1311-1319 (2000).

\section{Introduction}

Emphysema is estimated to affect 1.65 million people in the US (1). Emphysema is defined as abnormal permanent enlargement of the airspaces distal to terminal bronchioles. The pathogenesis of pulmonary emphysema, which is characterized by the disappearance of alveolar septa, remains enigmatic, although the protease-antiprotease imbalance hypothesis of inflammation is widely supported (2). Briefly, the concept is that activated alveolar macrophages release elastase, which destroys the lung tissue, overwhelming local antiprotease activities (3). However, because many cigarette smokers and patients with severe inflammatory lung parenchyma diseases (like pneumonia and adult respiratory distress syndrome) do not develop significant emphysema, this hypothesis may not fully explain the loss of lung tissue in cigarette smoking-induced emphysema.

Preceding the discovery of the association between panacinar emphysema and a hereditary deficiency of $\alpha$-1-antitrypsin (4), A.A. Liebow (5), based on histological examination of emphysema lungs, pointed out that the alveolar septa in centrilobular emphysema appear to be remarkably thin and almost avascular. He also considered that a reduction in the blood supply of the small precapillary blood vessels might induce the disappearance of alveolar septa. We revisit this early vascular hypothesis of emphysema development and propose that the disappearance of lung tissue in emphysema may involve the progressive loss of capillary endothelial and epithelial cells through the process of programmed cell death, apoptosis (6). One known survival factor for endothelial cells is VEGF, initially characterized as a factor that increases endothelial permeability (7) and induces endothelial cell growth (8). It is not only essential for the normal development of blood vessels in the embryo $(9,10)$, but it is also required for survival of endothelial cells. Withdrawal of VEGF leads to endothelial cell apoptosis in vitro $(11,12)$ and in vivo $(13)$. VEGF binds to two tyrosine kinase receptors present on endothelial cells: VEGF receptor 1 (VEGFR-1; Flt-1) and VEGFR-2 (KDR/Flk-1). Although VEGF is highly abundant in the lung, its normal biological activity in the lung is not well understood. We postulate that VEGF signaling may be required for the maintenance of adult lung alveolar structures. We have recently reported increased septal cell death in human emphysematous 
lungs, which was associated with reduced lung expression of VEGF and VEGFR-2 (KDR/Flk-1) (6). Here we report that chronic treatment of rats with the VEGF receptor blocker SU5416 causes alveolar cell apoptosis-dependent emphysema.

\section{Methods}

Animals. The animal protocol was approved by the Animal Care and Use Committee of the University of Colorado Health Sciences Center. Male Sprague-Dawley rats were purchased from a commercial vendor and kept for 7 days in the Animal Care Facility of the University of Colorado Health Sciences Center. In the first set of experiments, rats then were divided into three groups: (a) control group $(n=6)$, (b) SU5416 \{3-[(2,4-dimethylpyrrol-5-yl)methylidenyl]-indolin 2-one\} group $(n=6)$, and (c) SU5416+caspase inhibitor group $(n=6)$. SU5416 (provided by SUGEN Inc.) and SU5416+caspase inhibitor groups were injected subcutaneously three times per week for 3 weeks. SU5416 $(20 \mathrm{mg} / \mathrm{kg})$ was suspended in diluent ( $0.5 \%$ carboxymethylcellulose sodium, $0.9 \%$ sodium chloride, $0.4 \%$ polysorbate 80 , $0.9 \%$ benzyl alcohol in deionized water). The control group received only the diluent. SU5416+caspase inhibitor group was injected intraperitoneally with $1 \mathrm{mg}$ of Z-Asp- $\mathrm{CH}_{2}$-DCB (Alexis Corp., San Diego, California, USA), dissolved in $50 \mu \mathrm{l}$ of PBS solution containing $20 \%$ DMSO, every day for 3 weeks. Control and SU5416alone groups were injected only with $50 \mu \mathrm{l}$ of PBS solution containing $20 \%$ DMSO. To study the time course of the effect of SU5416, rats $(n=6)$ were treated for 3, 7, and 21 days.

Tissue processing. After completion of the treatment period, the chest was opened and the cardiopulmonary block was quickly isolated and excised, and the ratio of right ventricular weight/left ventricle plus septum weight was obtained. The right main bronchus was cross-clamped and the left lung was filled with $0.5 \%$ low melting agarose in $10 \%$ formalin at a constant pressure of $25 \mathrm{~cm} \mathrm{H}_{2} \mathrm{O}$, allowing for homogenous expansion of lung parenchyma (14). After that, the lungs were fixed in $10 \%$ formalin for 48 hours. The paraffin-embedded tissues were sectioned and prepared for histological analysis. Lung sections were taken from the same lung regions in both the treated and control groups, representative of upper and lower lobes of the left lung.

Morphological assessment. After fixation, $5-\mu \mathrm{m}$ sections were stained with hematoxylin and eosin. The mean linear intercept, as a measure of interalveolar wall distance, was determined by light microscopy at a total magnification of $\times 100$. The mean linear intercept was obtained by dividing the total length of a line drawn across the lung section by the total number of intercepts encountered in 72 lines per each rat lung, as described by Thurlbeck (15).

Angiograms. Arteriograms were performed in lungs that were infused with barium sulfate (16). Immediately after sacrifice, PBS was infused through a main pulmonary artery catheter to flush the pulmonary circulation free of blood. A barium sulfate-gelatin mixture was heated to $65^{\circ} \mathrm{C}$, and infused into the main pulmonary artery at a perfusion pressure of $74 \mathrm{mmHg}$. This pressure was maintained for at least 5 minutes to ensure penetration of the barium mixture. Lung tissue was subsequently fixed for angiograms. The quantitation of the peripheral pulmonary arteries filled with barium was performed as described recently (17).

Immunohistochemistry. Anti-caspase 3 polyclonal antibody CM1 (1:250 dilution; kindly provided by Anu Srinivasan, Idun Pharmaceuticals, La Jolla, California, USA) (18), proliferating cell nuclear antigen (PCNA; 1:50 dilution; sc-56 from Santa Cruz Biotechnology Inc., Santa Cruz, California, USA), and anti-macrophage antibody (1:50 dilution for immunohistochemistry; Chemicon International, Temecula, California, USA) were used. Immunolocalization was performed on paraffin-embedded, formalin-fixed rat lungs. Briefly, after paraffin removal in xylene, the sections were rehydrated and submitted to microwave treatment $(800 \mathrm{~W} / 15 \mathrm{~min})$ in $10 \mathrm{mM}$ citric acid monohydrate solution. After quenching of endogenous peroxidase with $3 \% \mathrm{H}_{2} \mathrm{O}_{2}$ for 20 minutes, the sections were exposed to CM1 polyclonal antibody, which recognizes active caspase- 3 , or to PCNA monoclonal antibody for 30 minutes. After incubation with the primary antibody, immunodetection was performed using biotinylated anti-rabbit or anti-mouse IgG. Peroxidase-conjugated streptavidin (Vector Laboratories, Burlingame, California, USA), with diaminobenzidine (DAB) as the substrate, completed the immunostaining. Negative controls for nonspecific binding included normal rabbit or mouse serum.

TUNEL. Terminal deoxynucleotidyl transferase-mediated dUTP nick end-labeling (TUNEL) was performed with TACS 2 TdT DAB kit (Trevigen, Gaithersburg, Maryland, USA), following the manufacturer's instructions. Briefly, after deparaffinization and rehydration, sections were digested with proteinase $\mathrm{K}$ at a concentration of $20 \mu \mathrm{g} / \mathrm{ml}$ for 15 minutes. Endogenous peroxidase activity was quenched with $2 \% \mathrm{H}_{2} \mathrm{O}_{2}$ for 5 minutes. The slides were immersed in terminal deoxynucleotidyl transferase (TdT) buffer. TdT, $1 \mathrm{mM} \mathrm{Mn}^{2+}$, and biotinylated dNTP in TdT buffer were then added to cover the sections and incubated in a humid atmosphere at $37^{\circ} \mathrm{C}$ for 60 minutes. The slides were washed with PBS and incubated with streptavidin-horseradish peroxidase for 10 minutes. After rinsing with PBS, the slides were immersed in DAB solution. The slides were counterstained for 3 minutes with $1 \%$ methyl green.

In situ ligation of labeled DNA fragments. A 200-bp double-stranded DNA fragment was prepared by polymerase chain reaction with Taq polymerase using primers 5'-CACTAGAGACAGTTTGCCATG-3' and 5'-GTCCCACACCCTTTAGAGAAG-3' complementary to human adenocarcinoma DNA. Deparaffinized $5-\mu \mathrm{m}$ sections were subjected to Taq polymerase-based DNA in situ ligation assay using the DNA fragments labeled with 
digoxigenin (DIG) according to the method described by Didenko and Hornsby (19). Briefly, sections were incubated for 90 minutes at $65^{\circ} \mathrm{C}$ in $10 \mathrm{mM}$ sodium citrate, $\mathrm{pH}$ 6.0, and then washed with water. Sections were incubated with $50 \mu \mathrm{g} / \mathrm{ml}$ proteinase $\mathrm{K}$ in PBS for 15 minutes. The DIG-labeled DNA fragments at $2 \mu \mathrm{g} / \mathrm{ml}$ and DNA T4 ligase (New England BioLabs, Beverly, Massachusetts, USA) at $10,000 \mathrm{U} / \mathrm{ml}$ in $50 \mathrm{mM}$ Tris$\mathrm{HCl}, \mathrm{pH}$ 7.8, $10 \mathrm{mM} \mathrm{MgCl}$, 10 mM DTT, 1 mM ATP, 25 $\mu \mathrm{g} / \mathrm{ml} \mathrm{BSA}, 15 \%$ polyethylene glycol then were added onto the slides. Sections were placed in a humidified box for 16 hours. The sections were washed with several changes of water over 2 hours. Sections labeled with DIG were detected using DIG nucleic acid detection kit and color solution (NBT/BCIP; Boehringer Mannheim Biochemicals Inc., Indianapolis, Indiana, USA).

Quantitation of proliferating alveolar septal cells. The percentage of PCNA-positive alveolar septal cells was determined by calculating (no. of PCNA-positive cells $/ 1000$ cells) $\times 100$, in five representative lungs from each animal group.

Lung tissue homogenates. The right lung was homogenized immediately after isolation in a buffer containing $50 \mathrm{mM}$ HEPES, pH 7.4, 0.1\% CHAPS, 1 mM DTT, $0.1 \mathrm{mM}$ EDTA, and $0.1 \%$ Triton X-100. Lung tissue homogenates were centrifuged at $10,000 \mathrm{~g}$ for $15 \mathrm{~min}-$ utes, and then supernatants were stored at $-70^{\circ} \mathrm{C}$ until they were used for Western blot analysis or caspase 3-like activity assay. Direct protein quantitation was by the $\mathrm{D}_{\mathrm{c}}$ protein assay kit (Bio-Rad, Laboratories Inc., Hercules, California, USA).

Caspase 3-like activity assay. The cytosolic extract was incubated at $37^{\circ} \mathrm{C}$ with $200 \mu \mathrm{M}$ substrate Ac-DEVDpNA in 96-well microtiter plates (Caspase 3 Cellular Activity Assay Kit Plus; Biomol Research Laboratories Inc., Plymouth Meeting, Pennsylvania, USA). At different time points, hydrolytic activities were determined by measuring the absorbance of $p$-nitroaniline at $405 \mathrm{~nm}$.

Detection of nucleosomal ladders. The presence of internucleosomal DNA cleavage was investigated with Apoalert LM-PCR Ladder Assay Kit (CLONTECH Laboratories Inc., Palo Alto, California, USA). In brief, DNA was isolated from lung tissue samples using the Wizard Plus SV Minipreps DNA Purification System (Promega Corp., Madison, Wisconsin, USA) according to the manufacturer's instructions. Twenty-five nanograms of purified DNA was used for LM-PCR assay. Dephosphorylated adaptors were ligated to $5^{\prime}$ phosphorylated blunt ends with T4 DNA ligase (for 16 hours at $16^{\circ} \mathrm{C}$ ). They then served as primers in LM-PCR under the following conditions: hot start ( $72^{\circ} \mathrm{C}$ for 8 minutes), 25 cycles $\left(94^{\circ} \mathrm{C}\right.$ for 1 minute and $72^{\circ} \mathrm{C}$ for 3 minutes), and postcycling $\left(72^{\circ} \mathrm{C}\right.$ for 15 minutes). Amplified DNA was subjected to gel electrophoresis on a $1.2 \%$ agarose gel containing ethidium bromide. The reaction set included thymus DNA
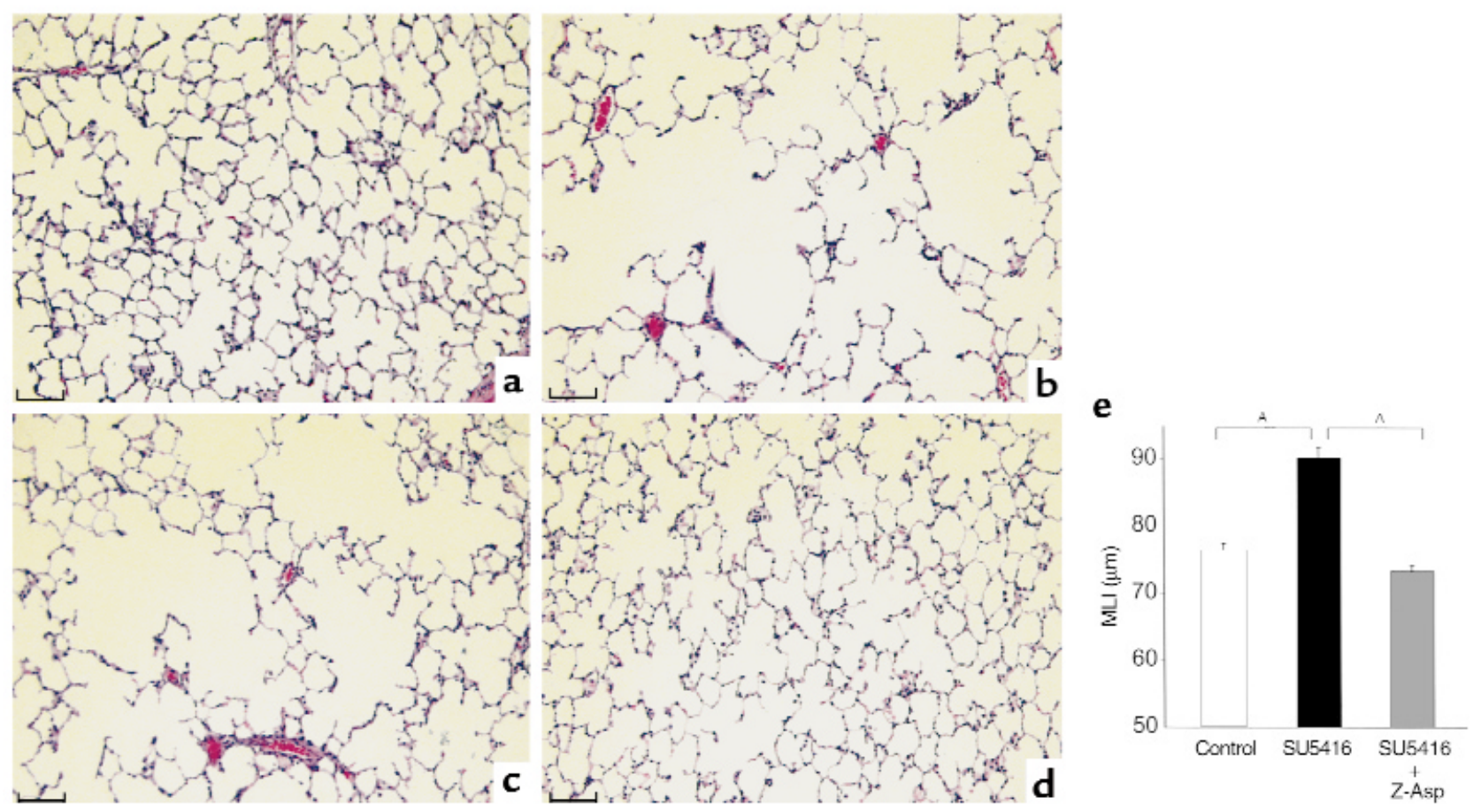

Figure 1

Histology of rat lungs. (a) Section of lung from a control rat showing normal alveolar structure. (b and c) Sections of lungs from an SU5416treated rat showing enlarged airspaces. (d) Section of lung from a rat treated with SU5416 plus Z-Asp-CH $\mathrm{C}_{2}$-DCB (caspase inhibitor) showing normal alveolar structure. Hematoxylin-and-eosin staining was used. Scale bars, $100 \mu \mathrm{m}$. (e) Emphysema in SU5416-treated lungs assessed by mean linear intercept. There were significantly greater mean linear intercept values in the SU5416-treated rat lungs $(n=6)$ when compared with the lungs from control rats $(n=6)$. Treatment with Z-Asp- $\mathrm{CH}_{2}-\mathrm{DCB}(n=6)$ prevented the increase in mean linear intercept in SU5416-treated rats. ${ }^{A} P<0.05$ (one-factor ANOVA, Scheffe F-test). 
a

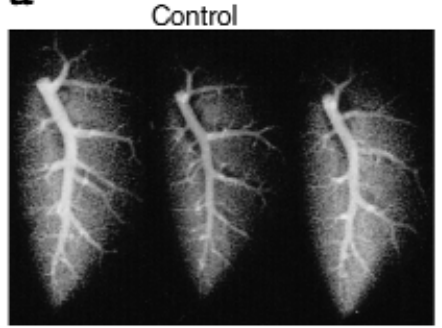

SU5416

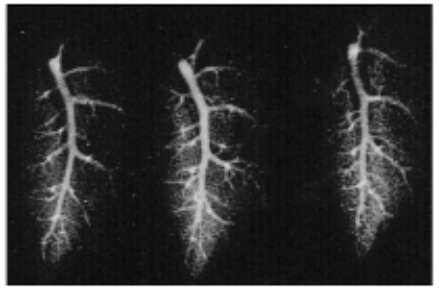

b

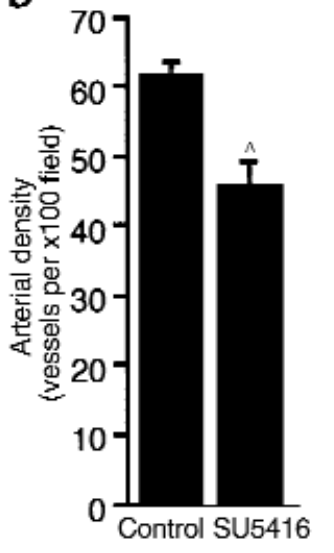

Figure 2

(a) Barium-gelatin angiograms of the left lungs of three SU5416-treated rats (below) compared with three control rat lungs (above). Barium filling was reduced in SU5416-treated rat lungs compared with control rat lungs. (b) Peripheral arterial density quantification of control and SU5416-treated lungs ( $\left.{ }^{A} P<0.05\right)$.

(25 ng) as positive control and for normalization of the amount of reaction product. Images were scanned, and the DNA fragmentation levels based on the density of the oligonucleosomal fragments were normalized relative to thymus control DNA.

Rat type II epithelial cell survival studies. Primary cultures of rat type II epithelial cells were isolated and cultured in Matrigel-coated 24-well plates as previously described (20). Duplicate wells were treated with SU5416 $(0.5,5$, or $50 \mu \mathrm{M})$ in $5 \%$ DMSO plus $1 \%$ Tween- 80 from a $500 \times$ stock or vehicle for 6 and 24 hours. The final concentration of DMSO in the medium was $0.01 \%$. Floating and attached cells were harvested at the specified time points and the DNA extracted as described above for lung tissue. The presence of internucleosomal DNA cleavage was investigated with Apoalert LM-PCR Ladder Assay Kit and quantitated as described above.

Western blot analysis and immunoprecipitation studies. Proteins $(25 \mathrm{mg}$ ) were subjected to electrophoresis on 4-12\% gradient Bio-Tris gels (Novex, San Diego, California, USA) and transferred to PolyScreen PVDF Transfer Membrane (NEN Life Science Products Inc., Boston, Massachusetts, USA) in Tris-glycine buffer containing 10\% methanol. Prestained molecular mass marker proteins (Bio-Rad Laboratories Inc.) were used as standards for the SDS-PAGE. Western blots were visualized using Renaissance Western Blot Chemiluminescence Reagent (NEN Life Science Products Inc.). Immunoprecipitation was performed according to the manufacturer's instructions (Santa Cruz Biotechnology Inc.). Antibodies used were: Flk-1 (A-3, 1:1000 dilution) (Santa Cruz Biotechnology Inc.), PI3 kinase (1:1000), Akt-1 (1:1000), phos-

photyrosine (1:1000) (Upstate Biotechnology Inc., Lake Placid, New York, USA), $\beta$-actin, and anti-sheep and anti-mouse HRP-conjugated (Sigma Chemical Co., St. Louis, Missouri, USA) and goat anti-rabbit HRP-conjugated antibodies (BioSource International, Camarillo, California, USA).

Statistical analysis. All the data are expressed as mean \pm SEM. Statistical analysis was performed with the Statview SE software package (Abacus Concepts Inc., Berkeley, California, USA). The data were normally distributed, and values obtained in the different groups of rats were compared using one-way ANOVA. Statistical difference was accepted at $P<0.05$.

\section{Results}

Histological studies. We initially tested in rats the effect of SU5416, a potent and selective inhibitor of VEGFR1 and -2 kinase activity (21). The lung weights of SU5416-treated rats were similar to those of control rats. Compared with control rat lungs, histological sections of the SU5416-treated rat lungs had enlarged airspaces (Figure 1, a-d). The mean linear intercept, as a measure of interalveolar wall distance, was significantly greater in SU5416-treated rats $(90.2 \pm 1.5 \mu \mathrm{m}$, Figure 1e) when compared with control rat lungs $(76.4 \pm 1.1 \mu \mathrm{m})$. The greater mean linear intercept in SU5416-treated rat lungs indicated that they were emphysematous, i.e., there was a loss of alveolar septa. This process was characterized by absence of increased infiltration by inflammatory cells as assessed by light microscopic examination of hematoxylin and eosin-stained slides and antimacrophage immunostaining, or fibrosis in SU5416-treated rat lungs when compared with control lungs. Microscopic examination of heart, liver, spleen, and bone marrow did not reveal any apparent histological abnormalities. a

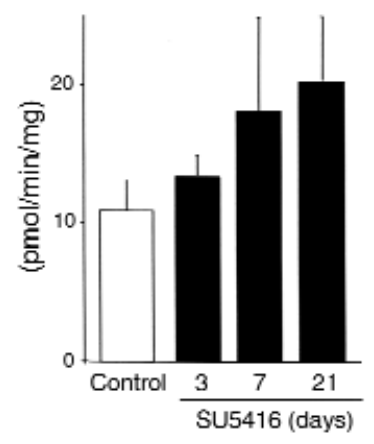

b

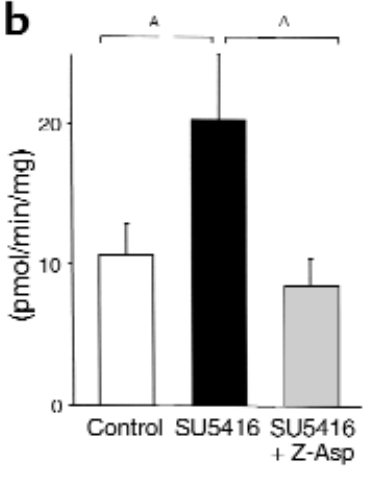

Figure 3

(a) Caspase 3-like activity in lungs during the SU5416 treatment. SU5416 treatment led to progressive increase of caspase 3-like activity in the rat lungs. (b) Comparison of caspase 3-like activity in lungs after 3 weeks of treatment. Caspase 3-like activity in SU5416-treated rat lungs $(n=6)$ was significantly higher than that of control rat lungs $(n=6)$, whereas lungs from rats treated with SU5416 $+\mathrm{Z}-\mathrm{Asp}-\mathrm{CH}_{2}-$ $\mathrm{DCB}(n=6)$ showed no increase in apoptotic activity. ${ }^{\mathrm{A}} P<0.05$ (onefactor ANOVA, Fisher's protected least significant difference test). 


\section{Figure 4}

Immunohistochemistry for active caspase 3 counterstained with methyl green. (a) Control rat lung, showing specific staining for caspase 3 in the alveolar septa (arrow). (b) SU5416treated rat lung, showing an increase of positive cells in the alveolar septa (arrow). (c) SU5416 plus Z-Asp- $\mathrm{CH}_{2-}$ DCB-treated rat lung. (d) SU5416treated rat lung. Positive cells (arrow) are observed in the endothelial cell layer of the pulmonary artery. Original magnifications, $\mathbf{a}-\mathbf{d}: \times 600$.

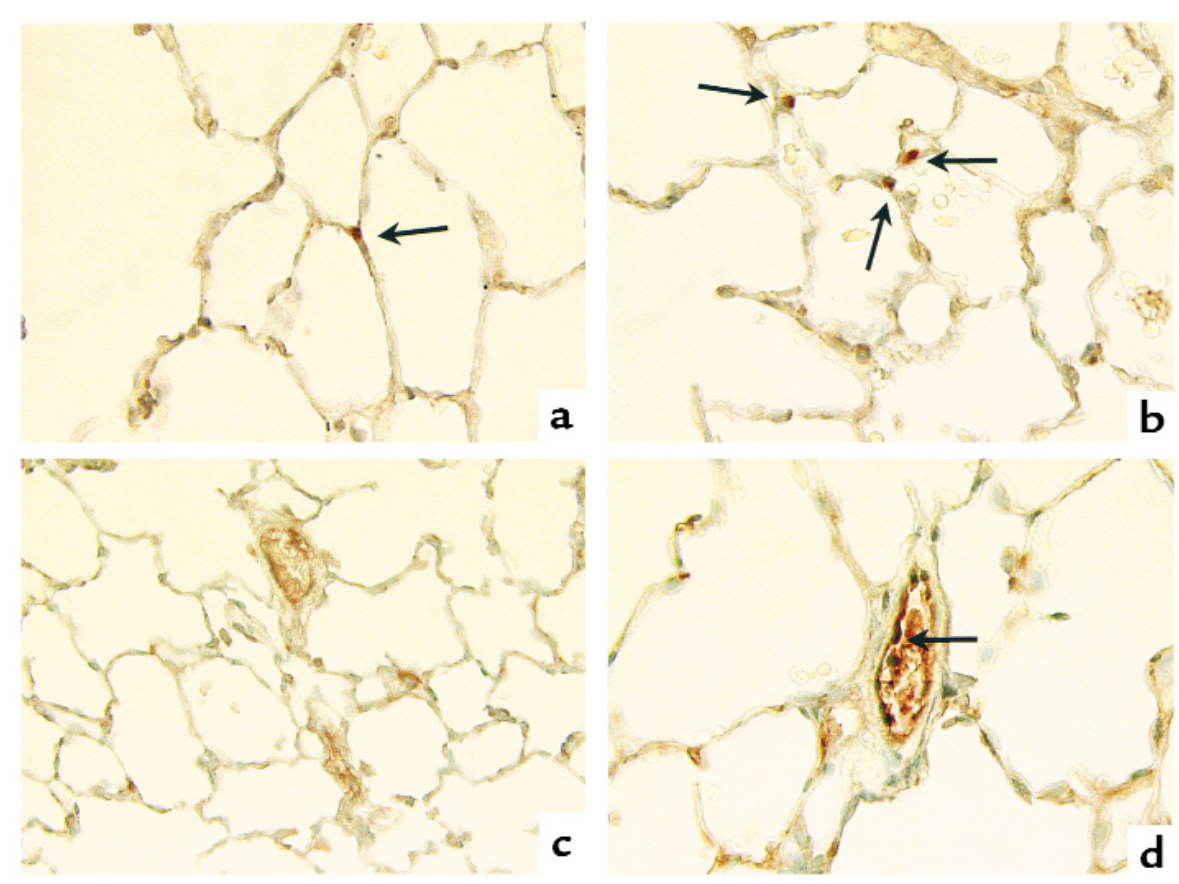

Angiograms. To examine the pulmonary vascular tree, we infused a barium-gelatin mixture into the main pulmonary artery of rats. Angiograms of the lungs showed a striking reduction in barium filling in SU5416-treated rats compared with control rats, consistent with pruning of the pulmonary artery tree in SU5416-treated rats (Figure 2a). The SU5416-treated emphysematous lungs had significantly fewer barium-filled peripheral pulmonary arteries than did control lungs (Figure $2 \mathrm{~b}$ ).

Apoptosis assays. To examine whether SU5416 caused alveolar cell apoptosis in vivo, we assayed for caspase 3-like activity. SU5416 treatment led to a progressive increase of caspase 3 -like activity in the lungs (13.5 \pm 1.5 , $18.1 \pm 6.9$, and $20.4 \pm 4.6 \mathrm{pmol} / \mathrm{min} / \mathrm{mg}$ on days 3,7 , and 21 , respectively, when compared with $10.7 \pm 2.2$ of the control; Figure 3a). Caspase 3-like activity in the SU5416-treated rat lungs after 3 weeks of treatment was significantly higher than that of the vehicle-treated control rat lungs $(20.4 \pm 4.6$ vs. $10.7 \pm 2.2 \mathrm{pmol} / \mathrm{min} / \mathrm{mg}$, $P<0.05$; Figure 3b).

Cells expressing processed or active caspase 3 are likely undergoing apoptosis (18). Active caspase 3 immunos- taining was detected in intra-alveolar, septal, and endothelial cells in both SU5416-treated and control rat lungs (Figure 4). There was an increase in the number of active caspase 3-positive alveolar cells per alveolar septal length in SU5416-treated rat lungs $(5.8 \pm 0.7$ per millimeter) when compared with control rat lungs ( $3.5 \pm 0.3$ per millimeter). SU5416 treatment did not result in reduction of alveolar cell proliferation, since similar numbers of PCNA-positive alveolar septal cells were observed in both SU5416-treated (24.7 $\pm 2.6 \%)$ and control rat lungs $(23.7 \pm 2.4 \%)$ (Figure 5).

The TUNEL technique was also used to detect apoptosis. Labeling of DNA strand breaks in situ by TUNEL demonstrated positive cells that were localized to the peribronchiolar, intra-alveolar, and septal cells in both SU5416-treated and control rat lungs, with increased septal cell apoptosis in the SU5416-treated rat lungs (Figure 6).

In situ ligation of labeled DNA fragments detects specifically double-strand DNA breaks with singlebase 3' overhangs in cells undergoing apoptosis (19). This assay is less sensitive but more specific for apop-

\section{Figure 5}

Immunohistochemistry for PCNA counterstained with hematoxylin, showing positive staining (brown) for proliferating cells in the intraalveolar space and alveolar septa. (a) Control rat lungs. (b) SU5416-treated rat lungs. Original magnifications, $\mathbf{a}$ and $\mathbf{b}: \times 400$
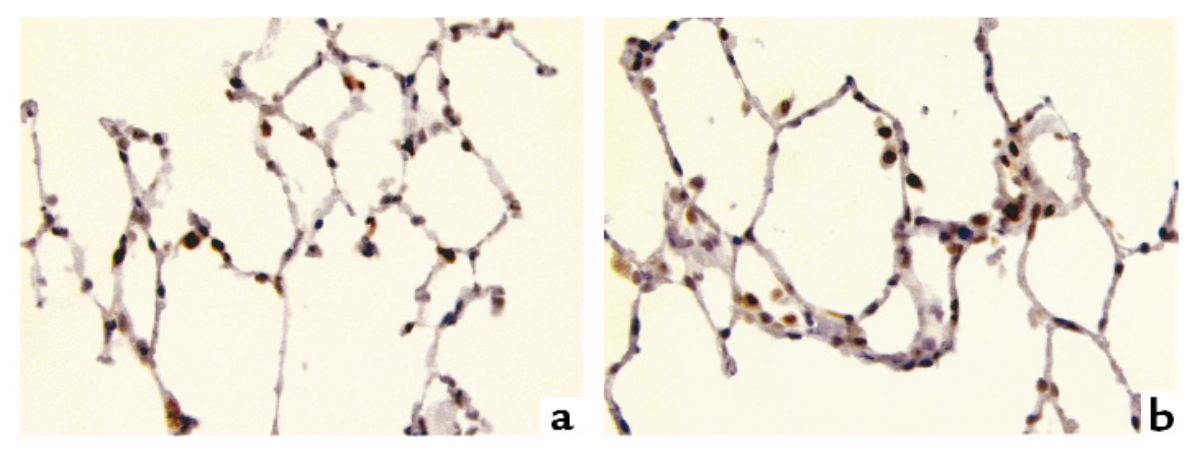


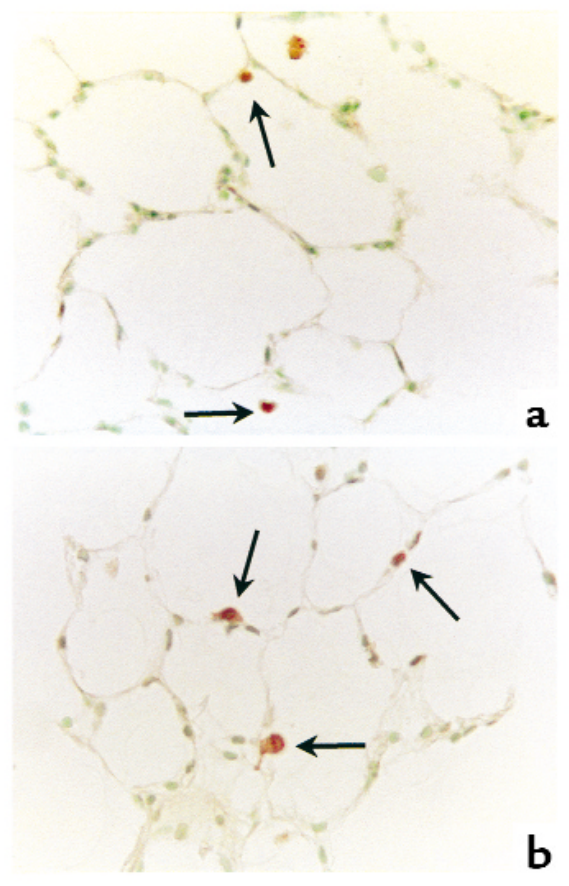

Figure 6

Lung sections stained by the TUNEL technique, counterstained with methyl green. (a) Control rat lungs, showing specific staining for DNA strand breaks in the intra-alveolar cells (arrows). (b) SU5416-treated rat lungs show abundant staining of cells in the alveolar septa (arrows). Original magnifications, $\mathbf{a}$ and $\mathbf{b}: \times 400$.

tosis than TUNEL. Although positive reactions were observed in alveolar cells in both SU5416-treated and control rat lungs (Figure 7), the lung tissue samples obtained from SU5416-treated rats showed increased apoptotic events compared with lung samples from control rats. The presence of apoptosis in SU5416treated lungs was confirmed by the detection of internucleosomal DNA ladders (Figure 8). Control lungs had evidence of low levels of DNA laddering, which is consistent with the in situ observation of levels of apoptosis in normal alveolar septal cells by TUNEL, in situ ligation of labeled DNA fragment labeling, or active caspase 3 immunostaining.

SU5416 treatment of primary cultures of rat type II epithelial cells. To determine whether the VEGF receptor blocker might cause rat type II epithelial cell apoptosis, we treated rat type II epithelial cells cultured onto Matrigel-coated wells with concentrations of SU5416 (of 0.5, 5, and $50 \mu \mathrm{M}$ ), for 6 and 24 hours. Morphologically, the VEGF receptor blocker did not cause any evidence of type II epithelial cell cytotoxicity. Furthermore, only background internucleosomal DNA cleavage, i.e., that found in DMSO-treated cells, was detected in floating and Matrigel-attached cells treated with SU5416 at all concentrations and time points (Table 1).

Western blot analysis. To determine how SU5416 treatment affected lung VEGFR-2 signaling, we examined the amounts of phosphorylated (active)
VEGFR-2, PI3 kinase, and Akt-1 in lung extracts using immunoprecipitation and Western blot analysis. SU5416 treatment led to a slight decrease in lung expression of VEGFR-2 and phosphorylated VEGFR2 (Figure 9). The nadir of VEGFR-2 protein expression (day 3 of SU5416 treatment) coincided with the time of maximal apoptotic DNA laddering. The decrease in VEGFR-2 protein expression following receptor blockade is consistent with the observation of a homologous feedback loop in which VEGF induces VEGFR-2 expression in cultured endothelial cells (22). The finding of decreased levels of Akt in the complex with VEGFR-2 (11) is also consistent with the increase in apoptosis (or impaired cell survival) seen at day 3 of SU5416 treatment. PI3 kinase levels in the complex appeared not to be altered.

Treatment with the caspase inhibitor. Because emphysema induced by SU5416 treatment was associated with increased lung apoptosis, we determined whether chronic treatment with the caspase inhibitor (Z-Asp$\mathrm{CH}_{2}$-DCB) would reduce the SU5416-associated apoptosis and emphysema. The antiapoptotic effect of Z-Asp- $\mathrm{CH}_{2}$-DCB was confirmed by the caspase 3-like activity assay. Caspase 3-like activity was increased in SU5416-treated rat lungs $(20.4 \pm 4.6 \mathrm{pmol} / \mathrm{min} / \mathrm{mg})$, whereas the lungs from rats treated with SU5416 plus $\mathrm{Z}$-Asp- $\mathrm{CH}_{2}$-DCB showed normal lung caspase 3-like activity $(8.6 \pm 1.9 \mathrm{pmol} / \mathrm{min} / \mathrm{mg}$; Figure 3$)$. The number of active caspase 3-immunopositive cells in the alveolar septa, normalized to the length of alveolar

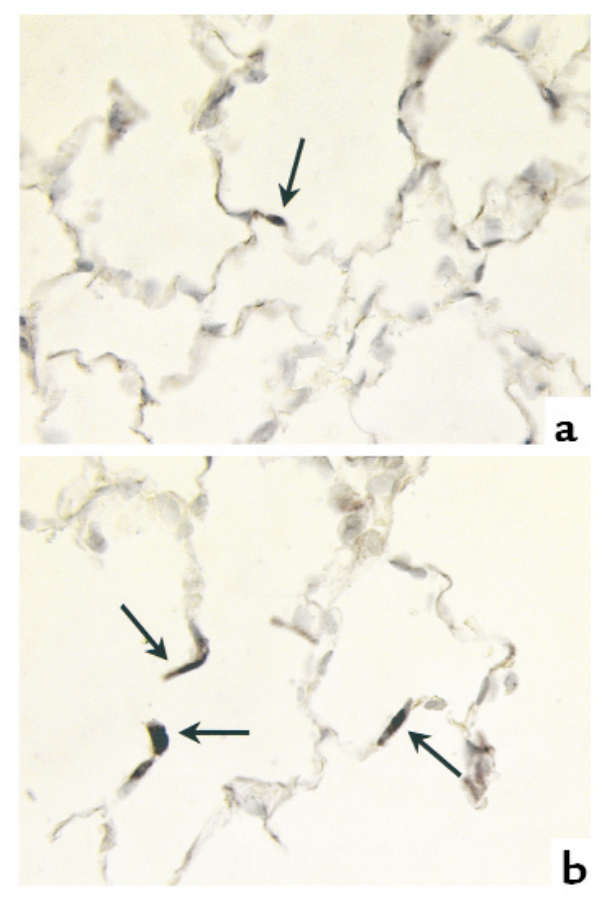

\section{Figure 7}

In situ ligation of labeled DNA fragments. (a) Control rat lung. (b) SU5416-treated rat lung. Positive reactions (arrows) were seen in alveolar septa. Original magnifications, $\mathbf{a}$ and $\mathbf{b}: \times 600$. 


\section{Figure 8}

(a) Detection of internucleosomal ladders using LM-PCR ladder assay. Internucleosomal ladders were observed in lung samples obtained from SU5416-treated rats. $M, 200$-bp DNA size markers; $P$, positive control calf thymus DNA; $\mathrm{H}$, no DNA in $\mathrm{H}_{2} \mathrm{O}$. Control samples consist of vehicle-treated lungs. (b) Densitometric units of internucleosomal DNA ladders in control $(n=2)$, and SU5416-treated lungs for $3(n=3), 7(n=3)$, and 21 days $(n=3)$. Results are shown as mean \pm SE and are significant at $P<0.01$ by the single-factor ANOVA. a

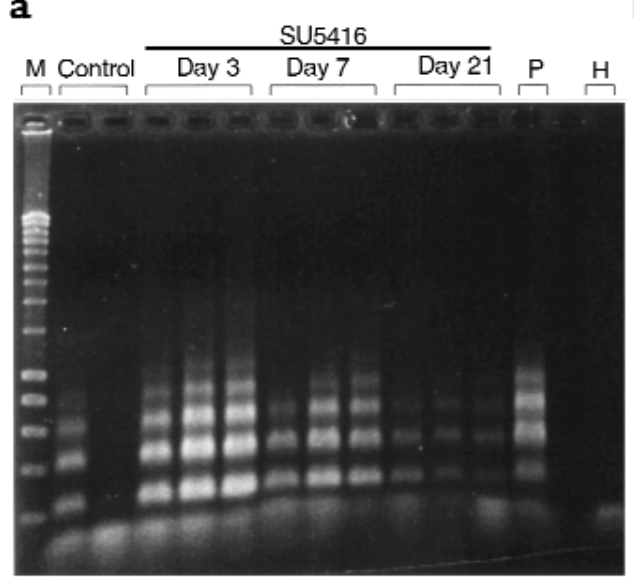

b

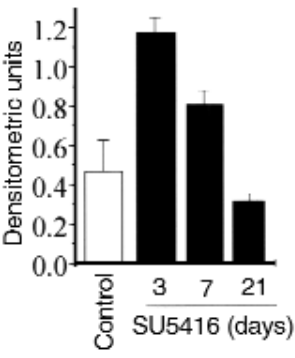

septa, was also decreased in the lungs treated with SU5416 plus Z-Asp- $\mathrm{CH}_{2}$-DCB $(2.4 \pm 0.3$ per millimeter) when compared with the lungs treated with SU5416 alone (5.8 \pm 0.7 per millimeter).

We also assessed whether the caspase inhibitor would prevent the emphysematous changes induced by the disturbance of the VEGFR-2 signaling pathway. Indeed, treatment with $\mathrm{Z}-\mathrm{Asp}-\mathrm{CH}_{2}-\mathrm{DCB}$ prevented the increase of the mean linear intercept in SU5416-treated rats $(73.0 \pm 0.8$ vs. $90.2 \pm 1.5 \mu \mathrm{m}, P<0.05$; Figure $1 \mathrm{e})$. By histological examination, the lungs from rats treated with the combination of SU5416 plus Z-Asp- $\mathrm{CH}_{2}-$ DCB had a normal appearance and were indistinguishable from vehicle-treated control rat lungs (Figure 1d). As shown in Figure 9, caspase inhibition with $\mathrm{Z}$-Asp- $\mathrm{CH}_{2}$-DCB had a remarkable effect in that it dramatically increased the amounts of PI3 kinase and Akt bound to VEGFR-2 protein. It is a novel finding that caspase inhibition can increase the in vivo expression of those kinases, which are involved in endothelial cell VEGF/VEGFR-2 survival signaling.

\section{Discussion}

This study shows - for the first time in an animal model, to our knowledge - that chronic blockade of VEGF receptors induces apoptosis in alveolar cells and that VEGF receptor signaling is required for the maintenance of the alveolar structures. These results are of interest because of the recent report of increased apoptosis and decreased VEGF protein in lungs from patients with smoking-induced emphysema (6). Because SU5416 increased endothelial cell apoptosis in colon cancer liver metastases (23), we hypothesized that impairment of the VEGF receptor kinase activity may induce endothelial cell apoptosis in the lung. Our finding of alveolar cell apoptosis and emphysema in the lungs of rats chronically treated with a VEGF receptor inhibitor is intriguing in view of the vascular hypothesis of emphysema presented by earlier investigators $(4,24,25)$. A second VEGF receptor blocker, structurally unrelated to SU5416, when administered to rats with an experi- mental protocol identical to that used for SU5416, confirmed our results of increased lung mean linear intercept as observed in rats treated with SU5416 (R.M. Tuder, unpublished observations).

The finding of emphysema in rats treated with SU5416 suggests that the VEGF receptor 2 (KDR/Flk-1) may play a more important role than VEGFR-1 (Flt-1) in the VEGF-mediated survival of lung capillary endothelial cells. The finding that newborn mice treated with a soluble Flt-Fc chimeric protein showed enlarged airspaces (26) very similar to those observed in rats treated with SU5416 further supports our hypothesis that blockade of VEGF survival signals in the lung causes emphysema.

On the other hand, additional actions of SU5416 in rat lungs may have contributed to alveolar cell apoptosis. When compared with control rats, SU5416 treatment caused an eightfold increase in lung isoprostane levels and a greater than 100 -fold induction of lung cytochrome P450 in the rat lungs (L. Alger et al., unpublished observations), which may catalyze the production of reactive oxygen species in pulmonary capillary endothelial cells and thus contribute to the apoptosis of the alveolar cells observed in SU5416-treated rat lungs. However, the findings that SU5416 treatment caused a decrease in lung tissue phosphorylated VEGFR-2 protein and a decrease

\section{Table 1}

Internucleosomal DNA laddering of rat type II epithelial cells attached in Matrigel or floating in the culture medium and treated with vehicle or SU5416 $(0.5,5,50 \mu \mathrm{M})$ for 6 and 24 hours

\begin{tabular}{lcccccc}
\hline & \multicolumn{3}{c}{ Matrigel } & \multicolumn{2}{c}{ Floating cells } \\
Compound & Dose & $6 \mathrm{~h}$ & $24 \mathrm{~h}$ & $6 \mathrm{~h}$ & $24 \mathrm{~h}$ \\
Vehicle & - & 1.0 & 0.80 & 0.79 & 0.73 \\
SU5416 & $0.5 \mu \mathrm{M}$ & 0.81 & 0.81 & 0.88 & 0.62 \\
SU5416 & $5 \mu \mathrm{M}$ & 0.88 & 0.88 & 0.88 & 0.68 \\
SU5416 & $50 \mu \mathrm{M}$ & 0.90 & 0.83 & 1.0 & 0.74
\end{tabular}

Results are the average of densitometric measurements in duplicate wells relative to the internucleosomal DNA laddering of thymus DNA (positive control), which was assigned a value of 1.0. 


\section{Figure 9}

(a) Expression of VEGFR-2 in lungs of rats treated with vehicle (control); SU5416 for 3, 7, or 21 days; or SU5416 + Z-Asp- $\mathrm{CH}_{2}$-DCB for 21 days. Each data point represents the average of expression in two lungs per experimental group. The bands were quantitated by densitometry, followed by normalization for loading and determination of protein integrity using $\beta$-actin expression. (b) Assessment of phosphorylated (active) VEGFR-2 in lungs of rats treated with vehicle (control); SU5416 for 3, 7, or 21 days; or SU5416 + Z-Asp-CH - -DCB for 21 days, by immunoprecipitation with anti-VEGFR-2 antibody and Western blot for VEGFR-2 and its phosphorylated form. Each data point represents the average of expression in two lungs per experimental group, and the data are expressed as the ratio of phosphorylated VEGFR-2 to nonphosphorylated VEGFR-2. (c) Assessment of Akt-1 in the complex with VEGFR-2 performed by immunoprecipitation with anti-VEGFR-2 antibody, and Western blot for VEGFR-2 and Akt-1. Data are expressed as the ratio of Akt-1 to VEGFR-2. (d) Assessment of PI3 kinase in the complex with VEGFR2 performed by immunoprecipitation with anti-VEGFR-2 antibody, and Western blot for VEGFR-2 and PI3 kinase. Data are expressed as the ratio of PI3 kinase to VEGFR-2. a

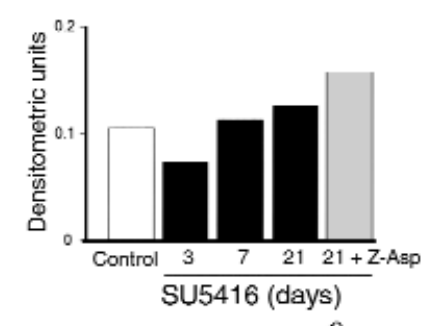

b $\frac{\text { SU5416 (days) }}{0.3 \quad 2121+\text { Z-Asp }}$ - - Phopho VEGFR-2 wart $=$ VEGFR-2

$\mathbf{C}$
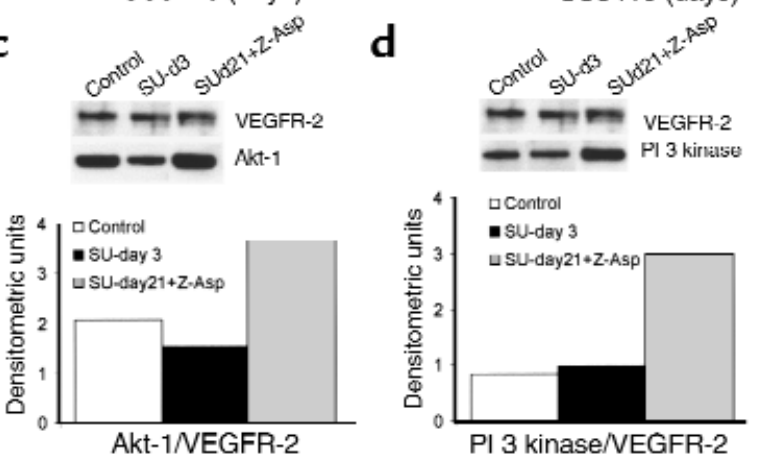

in the level of Akt indicate that SU5416 indeed acted as an inhibitor of VEGF receptors. We found that epithelial cell death was not increased when cultured type II cells were exposed to the VEGF receptor inhibitor. Because of this experimental result and the fact that VEGF acts as a survival factor for endothelial cells, we believe that emphysema in this model is a consequence of endothelial cell death.

It is of interest that the PCNA lung immunohistochemistry showed that cell proliferation in SU5416treated rats was comparable to that in control rats. This finding indicates that the drug did not inhibit cell proliferation but preferentially induced apoptosis, which may indeed cause the disappearance of alveolar septal structures in emphysema. Early angiographic studies of patients with pulmonary emphysema showed a reduction in the number and density of small precapillary arteries (27), comparable to the loss of precapillary arterioles in our animal studies.

Several animal models of emphysema have been developed based on a variety of strategies; treatment with cadmium, neutrophil elastase (28), and endotoxin have been employed to generate experimental emphysema in rodents (29). Of note, we have previously observed that endotoxin treatment caused a dramatic reduction in the expression of VEGF in lungs (30). Recently, it has been shown that disruption of the klotho gene causes pulmonary emphysema in mice (31), suggesting that klotho gene expression is necessary to maintain pulmonary structure integrity during postnatal life. Whether klotho gene expression relates to VEGF signaling is unknown.

We showed that SU5416-induced emphysema is caspase-dependent, since a broad caspase inhibitor blocked the development of emphysema. We do not know whether SU5416-induced alveolar cell death activates lung proteases, which may participate in the destruction of the elastic framework of the lung. It is conceivable that alveolar endothelial and epithelial septal cell death contributes to the elastase/antielastase imbalance by reducing the amount of available antiprotease proteins, such as the secretory leukoprotease inhibitor (SLPI). SLPI is expressed in association with the amorphous elastin present in the extracellular matrix of the alveolar walls, in bronchi and bronchioles, and in blood vessels (32). In addition, activated caspases may trigger matrix protease activity. Conversely, degradation of matrix proteins by metalloproteases may further enhance the susceptibility of endothelial cells to apoptosis due to VEGF receptor blockade or due to FGF withdrawal (33). Metalloproteases enhance the reabsorption stage of the neovasculature in later stages of angiogenesis in vivo (34), and their expression strongly colocalizes with areas of apoptosis during metamorphosis (33).

Taken together, our findings highlight the importance of apoptosis in the pathogenesis of emphysema and suggest that inhibition of apoptosis may offer a new strategy for the treatment of emphysema.

\section{Acknowledgments}

The authors wish to thank Yasushi Hoshikawa, George Halley, and Lori Alger for technical help, and Robert Mason for his help with the rat type II epithelial cell culture studies. This work has been supported by the Hart Family Foundation for Emphysema Research; NIH grants to R.M. Tuder (1RO1 HL60195-01, 1RO1 HL66554-01) and N.F. Voelkel 
(1RO1 HL60913-01); the Shirley Kiner Witham Memorial Pulmonary Hypertension Research Fund; and the Deutsche Forschungsgemeinschaft to J. Waltenberger (SFB451, B1; SFB497, C1; and Schwerpunktprogramm 1069 "Angiogenese").

1. 1995. Standards for the diagnosis and care of patients with chronic obstructive pulmonary disease. American Thoracic Society. Am. J. Respir. Crit. Care Med. 152:S77-S121.

2. Snider, G.L. 1989. Chronic obstructive pulmonary disease: a definition and implications of structural determinants of airflow obstruction for epidemiology. Am. Rev. Respir. Dis. 140:S3-S8.

3. Hautamaki, R.D., Kobayashi, D.K., Senior, R.M., and Shapiro, S.D. 1997. Requirement for macrophage elastase for cigarette smokeinduced emphysema in mice. Science. 277:2002-2004.

4. Eriksson, S. 1965. Studies in alpha-1-antitrypsin. Acta Med. Scand. 177:1-85.

5. Liebow, A.A. 1959. Pulmonary emphysema with special emphasis to vascular changes. Am. Rev. Respir. Dis. 80:67-93.

6. Kasahara, Y., et al. 2000. Endothelial cell death and decreased expression of vascular endothelial growth factor and its receptor KDR/Flk-1 in smoking-induced emphysema. Am. J. Respir. Crit. Care Med. In press.

7. Senger, D.R., et al. 1983. Tumor cells secrete a vascular permeability factor that promotes accumulation of ascites fluid. Science. 219:983-985.

8. Neufeld, G., Cohen, T., Gengrinovitch, S., and Poltorak, Z. 1999. Vascular endothelial growth factor (VEGF) and its receptor. FASEB J. 13:9-22.

9. Carmeliet, P., et al. 1996. Abnormal blood vessel development and lethality in embryos lacking a single VEGF allele. Nature. 380:435-439.

10. Ferrara, N., et al. 1996. Heterozygous embryonic lethality induced by targeted inactivation of the VEGF gene. Nature. 380:439-442.

11. Gerber, H.P., et al. 1998. Vascular endothelial growth factor regulates endothelial cell survival through the phosphatidylinositol 3'. kinase/Akt signal transduction pathway: requirement for Flr/KDR activation. J. Biol. Chem. 273:30336-30343.

12. Gerber, H.P., Dixit, V., and Ferrara, N. 1998. Vascular endothelial growth factor induces expression of the antiapoptotic proteins BCL-2 and A1 in vascular endothelial cells. J. Biol. Chem. 273:13313-13316.

13. Alon, T., et al. 1995. Vascular endothelial growth factor acts as a survival factor for newly formed retinal vessels and has implications for retinopathy of prematurity. Nat. Med. 1:1024-1028.

14. Halbower, A.C., Mason, R.J., Abman, S.H., and Tuder, R.M. 1994. Agarose infiltration improves morphology of cryostat sections of lung. Lab. Invest. 71:149-153.

15. Thurlbeck, W.M. 1967. Measurement of pulmonary emphysema. Am. Rev. Respir. Dis. 95:752-764.

16. Le Cras, T.D., et al. 1999. Abnormal lung growth and the development of pulmonary hypertension in the Fawn-Hooded rat. Am. J. Physiol. 277:L709-L718.

17. Jakkula, M., et al. 2000. Inhibition of angiogenesis decreases alveolar- ization in the developing rat lung. Am. J. Physiol. Lung Cell. Mol. Physiol. 279:L600-L607.

18. Srinivasan, A., et al. 1998. In situ immunodetection of activated caspase-3 in apoptotic neurons in the developing nervous system. Cell Death Differ. 5:1004-1016.

19. Didenko, V.V., and Hornsby, P.J. 1996. Presence of double-strand breaks with single-base $3^{\prime}$ overhang in cells undergoing apoptosis but not necrosis. J. Cell Biol. 135:1369-1376.

20. Osanai, K., Mason, R.J., and Voelker, D.R. 1998. Trafficking of newly synthesized surfactant protein A in isolated rat alveolar type II cells. Am. J. Respir. Cell Mol. Biol. 19:929-935.

21. Fong, T.A., et al. 1999. SU5416 is a potent and selective inhibitor of the vascular endothelial growth factor receptor (Flk-1/KDR) that inhibits tyrosine kinase catalysis, tumor vascularization, and growth of multiple tumor types. Cancer Res. 59:99-106.

22. Shen, B.Q., et al. 1998. Homologous up-regulation of KDR/Flk-1 receptor expression by vascular endothelial growth factor in vitro. J. Biol. Chem. 273:29979-29985.

23. Shaheen, R.M., et al. 1999. Angiogenic therapy targeting the tyrosine kinase receptor for vascular endothelial growth factor inhibits the growth of colon cancer liver metastasis and induces tumor and endothelial cell apoptosis. Cancer Res. 59:5412-5416.

24. Issaksohn, K. 1871. Pathologisch-anatomische Verändeerungen der Lungengefässe beim Emphysem. Virchows Arch. A Pathol. Pathol. Anat. 53:466-469.

25. Cudkowicz, L., and Armstrong, J.B. 1953. The bronchial arteries in pulmonary emphysema. Thorax. 8:46-58.

26. Gerber, H.P., et al. 1999. VEGF is required for growth and survival in neonatal mice. Development. 126:1149-1159.

27. Cordasco, E.M., Beerel, F.R., Vance, J.W., Wende, R.W., and Toffolo, R.R. 1968. Newer aspects of the pulmonary vasculature in chronic lung disease. A comparative study. Angiology. 19:399-407.

28. Massaro, G.D., and Massaro, D. 1997. Retinoic acid treatment abrogates elastase-induced pulmonary emphysema in rats. Nat. Med. 3:675-677.

29. Shapiro, S.D. 2000. Animal models for chronic obstructive pulmonary diseases. Am. J. Respir. Cell Mol. Biol. 22:4-7.

30. Tuder, R.M., Flook, B.E., and Voelkel, N.F. 1995. Increased gene expression for VEGF and the VEGF receptors KDR/Flk and Flt in lungs exposed to acute or to chronic hypoxia. Modulation of gene expression by nitric oxide. J. Clin. Invest. 95:1798-1807.

31. Suga, T., et al. 2000. Disruption of the klotho gene causes pulmonary emphysema in mice. Defect in maintenance of pulmonary integrity during postnatal life. Am. J. Respir. Cell Mol. Biol. 22:26-33.

32. Willems, L.N., Otto-Verberne, C.J., Kramps, J.A., Ten, H.O., and Dijkman, J.H. 1986. Detection of antileukoprotease in connective tissue of the lung. Histochemistry. 86:165-168.

33. Kuzuya, M., et al. 1999. Induction of apoptotic cell death in vascular endothelial cells cultured in three-dimensional collagen lattice. Exp. Cell Res. 248:498-508.

34. Zhu, W.H., Guo, X., Villaschi, S., and Francesco, N.R. 2000. Regulation of vascular growth and regression by matrix metalloproteinases in the rat aorta model of angiogenesis. Lab. Invest. 80:545-555. 